Journal of Applied Mathematics and Stochastic Analysis, 16:3 (2003), 257-265.

Printed in the USA (C)2003 by North Atlantic Science Publishing Company

\title{
ON AN EVEN ORDER NEUTRAL DIFFERENTIAL INEQUALITY
}

\author{
PEIGUANG WANG \\ Hebei University \\ College of Electronic and Information \\ Baoding 071002, PR of China \\ E-mail: pgwang@mail.hbu.edu.cn \\ and \\ LOKENATH DEBNATH \\ University of Texas-Pan American ${ }^{1}$ \\ Department of Mathematics \\ Edinburg, TX 78539 USA \\ E-mail: debnath@panam.edu
}

(Received July, 2002; Revised November, 2002)

In this paper, we prove new results related to the nonexistence criteria for eventually positive solutions of certain even order neutral differential inequality with distributed deviating arguments.

Keywords: Differential Inequality, Neutral Type, Distributed Deviating Arguments, Eventually Postive Solution.

AMS (MOS) subject classification: 34K11, 34K40.

\section{Introduction}

In order to make this paper self-contained, we introduce the following definition.

Definition 1: The function $f(t)$ is said to be eventually zero if there exists a sufficiently large $t_{\mu}$ such that $f(t) \equiv 0$ holds for $t \geq t_{\mu}$.

This paper is concerned with nonexistence conditions of eventually positive solutions of the even order neutral differential inequality with distributed deviating arguments

$$
[x(t)+c(t) x(t-\tau)]^{(n)}+\int_{a}^{b} p(t, \xi) f(x[g(t, \xi)]) d \sigma(\xi) \leq 0, \quad t \geq t_{0},
$$

in which $\tau>0$ is a constant, $n$ is an even positive integer; $c(t) \in C(I, R), 0 \leq c(t) \leq 1$, and $p(t, \xi) \in C\left(I \times J, R_{+}\right)$is not eventually zero on any $I_{\mu} \times J, I=\left[t_{0}, \infty\right), J=[a, b]$,

\footnotetext{
${ }^{1}$ This work was supported by the Faculty Research Council of the University of Texas-Pan American
} 
$I_{\mu}=\left[t_{\mu}, \infty\right), t_{\mu} \geq t_{0}, R_{+}=[0, \infty)$. Furthermore, we assume that $g(t, \xi) \in C(I \times J, R)$ is nondecreasing with respect to $t$ and $\xi$, respectively, $\frac{d}{d t} g(t, a)$ exists, $g(t, \xi) \leq t$ for $\xi \in J$, and $\liminf _{t \rightarrow \infty, \xi \in J}\{g(t, \xi)\}=\infty ; f(x) \in C(R, R)$, and $x f(x)>0(x \neq 0) ; \sigma(\xi) \in(J, R)$ is nondecreasing; integral of inequality (1) is in Lebesgue-Stieltjes sense.

Recently, Li and Cui [1] have obtained some results dealing with a class of even order neutral differential inequalities with applications. On the other hand, Liu and $\mathrm{Fu}[2]$ have studied nonlinear differential inequality with distributed deviating arguments and their applications. These authors provided some results on nonexistence conditions of eventually positive solutions of inequality (1). For example,

Theorem A:(See [1]) If $0 \leq c(t) \leq 1$, and

$$
\int_{t_{0}}^{t} \int_{a}^{b} p(s, \xi)\{1-c[g(s, \xi)]\} d \sigma(\xi) d s=\infty
$$

then inequality (1) has no eventually positive solutions.

Theorem B:(See ([2]) Assume that $f(-x)=-f(x), x \in(0, \infty)$, and

$$
\frac{f(x)}{x} \geq \lambda, \quad x \in(0, \infty), \quad \text { for some constant } \quad \lambda>0 .
$$

If there exists a monotonically increasing function $\varphi(t) \in C^{\prime}(I,(0, \infty))$ such that

$$
\int_{t_{0}}^{t}\left[\lambda \varphi(s) \int_{a}^{b} p(s, \xi)\{1-c[g(s, \xi)]\} d \sigma(\xi)-A \varphi^{\prime}(s)\right] d s=\infty
$$

for any number $A>0$, then inequality (1) has no eventually positive solutions.

The purpose of this paper is to obtain two new results related to the nonexistence criteria for eventually positive solutions of inequality (1). In the established nonexistence criteria, there is a general class of function $H(t, s)$ as the parameter function. By choosing a different function $H(t, s)$, we are able to derive some useful corollaries.

Definition 2: The solution $x(t) \in C^{(n)}(I, R)$ of inequality (1) is said to be eventually positive if there exists a sufficiently large positive number $T \geq t_{0}$ such that the inequality $x(t)>0$ holds for $t \geq T$.

To develop the nonexistence criteria of eventually positive solutions of inequality (1), we first need the following Lemmas:

Lemma 1: (See [1]) Assume that $x(t)$ is an eventually positive solution of inequality (1). Let

$$
y(t)=x(t)+c(t) x(t-\tau) .
$$

Then there exists a $t_{1} \geq t_{0}$ such that

$$
y(t)>0, \quad y^{\prime}(t)>0, \quad y^{(n-1)}(t)>0 \text { and } y^{(n)}(t) \leq 0, \quad t \geq t_{1} .
$$

Lemma 2: (See [3]) Let $x^{(n)}(t) \in C\left(I, R_{+}\right)$. If $x^{(n)}(t)$ is eventually of one sign for all large $t$, and $x^{(n)}(t) \times x^{(n-1)}(t) \leq 0$ for $t_{1}>t_{0}$, then there exists a constant $\theta \in(0,1)$ such that for sufficiently large $t$, there exists a constant $M_{\theta}>0$ satisfying

$$
\left|u^{\prime}(t / 2)\right| \geq M_{\theta} t^{n-2}\left|u^{(n-1)}(t)\right| .
$$




\section{Main Results}

The following theorems provide sufficient conditions leading to nonexistence of eventually positive solutions for inequality (1).

Theorem 1: Assume that the condition of Theorem $B$ holds, and there exist functions $H(t, s) \in C^{\prime}(D ; R), h(t, s) \in C(D ; R)$, with $D=\left\{(t, s) \mid t \geq s \geq t_{0}\right\}$ satisfying

$\left(H_{1}\right) H(t, t)=0, t \geq t_{0} ; H(t, s)>0, t>s \geq t_{0} ;$

$\left(H_{2}\right) H_{t}{ }^{\prime}(t, s) \geq 0, H_{s}{ }^{\prime}(t, s) \leq 0$, and $-H_{s}{ }^{\prime}(t, s)=h(t, s) \sqrt{H(t, s)},(t, s) \in D$.

If

$$
\limsup _{t \rightarrow \infty} \frac{1}{H\left(t, t_{0}\right)} \int_{t_{0}}^{t} H(t, s) \int_{a}^{b} p(s, \xi)\{1-c[g(s, \xi)]\} d \sigma(\xi) d s=\infty
$$

and

$$
\limsup _{t \rightarrow \infty} \frac{1}{H\left(t, t_{0}\right)} \int_{t_{0}}^{t} \frac{h^{2}(t, s)}{g^{n-2}(s, a) g^{\prime}(s, a)} d s<\infty,
$$

then inequality (1) has no eventually positive solutions.

Proof: Assume to the contrary that $x(t)$ is an eventually positive solution of inequality (1). Then from $\lim _{t \rightarrow \infty}, \inf _{\xi \in J}\{g(t, \xi)\}=\infty$, there exists a $t_{1} \geq t_{0}$ such that $x(t)>0, x(t-\tau)>0$ and $x[g(t, \xi)]>0$ for $t \geq t_{1}$ and $\xi \in J$. From (2) and (3), inequality (1) can be written as

$$
\begin{aligned}
0 & \geq y^{(n)}(t)+\int_{a}^{b} p(t, \xi) f(x[g(t, \xi)]) d \sigma(\xi) \\
& \geq y^{(n)}(t)+\lambda \int_{a}^{b} p(t, \xi)\{y[g(t, \xi)]-c[g(t, \xi)] x[g(t, \xi)-\tau]\} d \sigma(\xi) .
\end{aligned}
$$

From Lemma $1, y^{\prime}(t)>0$ and $y(t) \geq x(t), t \geq t_{1}$, hence $y[g(t, \xi)] \geq y[g(t, \xi)-\tau] \geq$ $x[g(t, \xi)-\tau]$. Thus

$$
y^{(n)}(t)+\lambda \int_{a}^{b} p(t, \xi)\{1-c[g(t, \xi)]\} y[g(t, \xi)] d \sigma(\xi) \leq 0, \quad t \geq t_{1} .
$$

Furthermore, in view of $g(t, \xi)$ being nondecreasing with respect to $\xi$, we have

$$
y^{(n)}(t)+\lambda y[g(t, a)] \int_{a}^{b} p(t, \xi)\{1-c[g(t, \xi)]\} d \sigma(\xi) \leq 0, \quad t \geq t_{2} .
$$

Let

$$
z(t)=\frac{y^{(n-1)}(t)}{y\left[\frac{g(t, a)}{2}\right]} .
$$

Then $z(t) \geq 0$. Since $\frac{d}{d t} g(t, a)$ exists, we obtain $y^{\prime}[g(t, a)]=\frac{d y}{d g} \frac{d}{d t} g(t, a)$. Furthermore, from Lemma 1, $y^{(n)}(t) \leq 0$, and in view of $g(t, \xi)$ being nondecreasing with respect to $\xi$, $g(t, \xi) \leq t$ for $\xi \in J$, we obtain $y^{(n-1)}(t) \leq y^{(n-1)}[g(t, a)] \leq y^{(n-1)}\left[\frac{g(t, a)}{2}\right]$. Thus, from 
Lemma 2, we have

$$
\begin{aligned}
z^{\prime}(t) & =\frac{y^{(n)}(t)}{y\left[\frac{g(t, a)}{2}\right]}-\frac{1}{2} \frac{y^{(n-1)}(t) y^{\prime}\left[\frac{g(t, a)}{2}\right] g^{\prime}(t, a)}{y^{2}\left[\frac{g(t, a)}{2}\right]} \\
& \leq \frac{y^{(n)}(t)}{y\left[\frac{g(t, a)}{2}\right]}-\frac{M_{\theta}}{2} g^{n-2}(t, a) g^{\prime}(t, a) z^{2}(t),
\end{aligned}
$$

Furthermore, from $y^{\prime}(t)>0$ and $(8)$, for $t \geq t_{2}$, we obtain

$$
z^{\prime}(t) \leq-\lambda \int_{a}^{b} p(t, \xi)\{1-c[g(t, \xi)]\} d \sigma(\xi)-\frac{M_{\theta}}{2} g^{n-2}(t, a) g^{\prime}(t, a) z^{2}(t)
$$

Integrating by parts for any $t>T \geq t_{1}$, and using the properties $\left(H_{1}\right)$ and $\left(H_{2}\right)$, we have

$$
\begin{aligned}
& \lambda \int_{T}^{t} H(t, s) \int_{a}^{b} p(s, \xi)\{1-c[g(s, \xi)]\} d \sigma(\xi) d s \\
& \leq-\int_{T}^{t} H(t, s) z^{\prime}(s) d s-\frac{M_{\theta}}{2} \int_{T}^{t} H(t, s) g^{n-2}(s, a) g^{\prime}(s, a) z^{2}(s) d s \\
& =-\int_{T}^{t} H(t, s) d z(s)-\frac{M_{\theta}}{2} \int_{T}^{t} H(t, s) g^{n-2}(s, a) g^{\prime}(s, a) z^{2}(s) d s \\
& =H(t, T) z(T)-\int_{T}^{t} h(t, s) \sqrt{H(t, s)} z(s) d s \\
& -\frac{M_{\theta}}{2} \int_{T}^{t} H(t, s) g^{n-2}(s, a) g^{\prime}(s, a) z^{2}(s) d s \\
& =H(t, T) z(T)-\frac{1}{2} \int_{T}^{t}\left[\sqrt{M_{\theta} H(t, s) g^{n-2}(s, a) g^{\prime}(s, a)} z(s)\right. \\
& \left.+\frac{h(t, s)}{\sqrt{M_{\theta} g^{n-2}(s, a) g^{\prime}(s, a)}}\right]^{2} d s+\int_{T}^{t} \frac{h^{2}(t, s)}{2 M_{\theta} g^{n-2}(s, a) g^{\prime}(s, a)} d s,
\end{aligned}
$$

which implies that

$$
\begin{aligned}
& \int_{T}^{t}\left[\lambda H(t, s) \int_{a}^{b} p(s, \xi)\{1-c[g(s, \xi)]\} d \sigma(\xi)-\frac{h^{2}(t, s)}{2 M_{\theta} g^{n-2}(s, a) g^{\prime}(s, a)}\right] d s \\
& \leq H(t, T) z(T)-\frac{1}{2} \int_{T}^{t}\left[\sqrt{M_{\theta} H(t, s) g^{n-2}(s, a) g^{\prime}(s, a)} z(s)\right. \\
& \left.\quad+\frac{h(t, s)}{\sqrt{M_{\theta} g^{n-2}(s, a) g^{\prime}(s, a)}}\right]^{2} d s .
\end{aligned}
$$

Furthermore, in view of $\left(H_{2}\right)$, for $t_{1} \geq t_{0}$, we have $H\left(t, t_{1}\right) \leq H\left(t, t_{0}\right)$. Thus

$$
\begin{aligned}
& \int_{t_{1}}^{t}\left[\lambda H(t, s) \int_{a}^{b} p(s, \xi)\{1-c[g(s, \xi)]\} d \sigma(\xi)-\frac{h^{2}(t, s)}{2 M_{\theta} g^{n-2}(s, a) g^{\prime}(s, a)}\right] d s \\
& \leq H\left(t, t_{1}\right) z\left(t_{1}\right) \leq H\left(t, t_{0}\right) z\left(t_{1}\right)
\end{aligned}
$$




$$
\begin{aligned}
& \frac{1}{H\left(t, t_{0}\right)} \int_{t_{0}}^{t}\left[\lambda H(t, s) \int_{a}^{b} p(s, \xi)\{1-c[g(s, \xi)]\} d \sigma(\xi)-\frac{h^{2}(t, s)}{2 M_{\theta} g^{n-2}(s, a) g^{\prime}(s, a)}\right] d s \\
= & \frac{1}{H\left(t, t_{0}\right)}\left[\int_{t_{0}}^{t_{1}}+\int_{t_{1}}^{t}\right]\left[\lambda H(t, s) \int_{a}^{b} p(s, \xi)\{1-c[g(s, \xi)]\} d \sigma(\xi)\right. \\
& \left.-\frac{h^{2}(t, s)}{2 M_{\theta} g^{n-2}(s, a) g^{\prime}(s, a)}\right] d s \\
\leq & z\left(t_{1}\right)+\int_{t_{0}}^{t_{1}} \frac{H(t, s)}{H\left(t, t_{0}\right)} \int_{a}^{b} p(s, \xi)\{1-c[g(s, \xi)]\} d \sigma(\xi) d s \\
\leq & z\left(t_{1}\right)+\int_{t_{0}}^{t_{1}} \int_{a}^{b} p(s, \xi)\{1-c[g(s, \xi)]\} d \sigma(\xi) d s .
\end{aligned}
$$

It follows from (13) that

$$
\begin{gathered}
\limsup _{t \rightarrow \infty} \frac{1}{H\left(t, t_{0}\right)} \int_{t_{0}}^{t} \lambda H(t, s) \int_{a}^{b} p(s, \xi)\{1-c[g(s, \xi)]\} d \sigma(\xi) \\
\leq \quad \limsup _{t \rightarrow \infty} \frac{1}{H\left(t, t_{0}\right)} \int_{t_{0}}^{t}\left[\lambda H(t, s) \int_{a}^{b} p(s, \xi)\{1-c[g(s, \xi)]\} d \sigma(\xi)\right. \\
\left.\quad-\frac{h^{2}(t, s)}{2 M_{\theta} g^{n-2}(s, a) g^{\prime}(s, a)}\right] d s \\
\quad+\limsup _{t \rightarrow \infty} \frac{1}{H\left(t, t_{0}\right)} \int_{t_{0}}^{t} \frac{h^{2}(t, s)}{g^{n-2}(s, a) g^{\prime}(s, a)} d s \\
\leq \quad z\left(t_{1}\right)+\int_{t_{0}}^{t_{1}} \int_{a}^{b} p(s, \xi)\{1-c[g(s, \xi)]\} d \sigma(\xi) d s \\
\quad+\limsup _{t \rightarrow \infty} \frac{1}{H\left(t, t_{0}\right)} \int_{t_{0}}^{t} \frac{h^{2}(t, s)}{g^{n-2}(s, a) g^{\prime}(s, a)} d s \\
<\quad \infty, \quad
\end{gathered}
$$

which contradicts (4). Therefore, the proof of Theorem 1 is complete.

Remark 1: From Theorem 1, we can establish various sufficient conditions by means of the choices of parameter function $H(t, s)$. For example, choosing $H(t, s)=(t-s)^{m-1}$, $t \geq s \geq t_{0}$, in which $m>2$ is an integer, we obtain $h(t, s)=(m-1)(t-s)^{\frac{m-3}{2}}, t \geq s \geq t_{0}$. From Theorem 1, we have

Corollary 1: If there exists an integer $m>2$ such that

$$
\begin{gathered}
\limsup _{t \rightarrow \infty} \frac{1}{t^{m-1}} \int_{t_{0}}^{t}(t-s)^{m-1} \int_{a}^{b} p(s, \xi)\{1-c[g(s, \xi)]\} d \sigma(\xi) d s=\infty \\
\limsup _{t \rightarrow \infty} \frac{1}{t^{m-1}} \int_{t_{0}}^{t} \frac{(m-1)^{2}(t-s)^{m-3}}{g^{n-2}(s, a) g^{\prime}(s, a)} d s<\infty
\end{gathered}
$$

then inequality (1) has no eventually positive solutions.

If

$$
\limsup _{t \rightarrow \infty} \frac{1}{H\left(t, t_{0}\right)} \int_{t_{0}}^{t} H(t, s) \int_{a}^{b} p(s, \xi)\{1-c[g(s, \xi)]\} d \sigma(\xi) d s<\infty,
$$


we have the following result:

Theorem 2: Assume that the conditions of Theorem 1 and (16) hold. If $H_{t}^{\prime}(t, s)$ is nondecreasing, and there exists a function $\varphi(t) \in C(I, R)$ satisfying

$$
\begin{gathered}
\liminf _{t \rightarrow \infty} \frac{1}{H\left(t, t_{0}\right)} \int_{u}^{t}\left[\lambda H(t, s) \int_{a}^{b} p(s, \xi)\{1-c[g(s, \xi)]\} d \sigma(\xi)\right. \\
\left.-\frac{h^{2}(t, s)}{2 M_{\theta} g^{n-2}(s, a) g^{\prime}(s, a)}\right] d s \geq \varphi(u), u \geq t_{0}, \\
\lim _{t \rightarrow \infty} \frac{1}{H\left(t, t_{0}\right)} \int_{t_{0}}^{t} H(t, s) g^{n-2}(s, a) g^{\prime}(s, a) \varphi_{+}^{2}(s) d s=\infty, \quad \varphi_{+}(s)=\max _{s \geq t_{0}}\{\varphi(s), 0\}
\end{gathered}
$$

then inequality (1) has no eventually positive solutions.

Proof: Assume to the contrary that $x(t)$ is an eventually positive solution of inequality (1). Then from the proof of Theorem 1 , there exists a $t_{1} \geq t_{0}$ such that

$$
z^{\prime}(t) \leq-\lambda \int_{a}^{b} p(t, \xi)\{1-c[g(t, \xi)]\} d \sigma(\xi)-\frac{M_{\theta}}{2} g^{n-2}(t, a) g^{\prime}(t, a) z^{2}(t)
$$

Thus

$$
\begin{gathered}
\lambda \int_{t_{1}}^{t} H(t, s) \int_{a}^{b} p(s, \xi)\{1-c[g(s, \xi)]\} d \sigma(\xi) d s \\
\leq-\int_{t_{1}}^{t} H(t, s) z^{\prime}(s) d s-\frac{M_{\theta}}{2} \int_{t_{1}}^{t} H(t, s) g^{n-2}(s, a) g^{\prime}(s, a) z^{2}(s) d s \\
=\quad H\left(t, t_{1}\right) z\left(t_{1}\right)-\int_{t_{1}}^{t} \sqrt{H(t, s)} h(t, s) z(s) d s \\
-\frac{M_{\theta}}{2} \int_{t_{1}}^{t} H(t, s) g^{n-2}(s, a) g^{\prime}(s, a) z^{2}(s) d s
\end{gathered}
$$

and

$$
\begin{gathered}
\lambda \int_{t_{1}}^{t} H(t, s) \int_{a}^{b} p(s, \xi)\{1-c[g(s, \xi)]\} d \sigma(\xi) d s \\
\leq \quad H\left(t, t_{1}\right) z\left(t_{1}\right)-\frac{1}{2} \int_{t_{1}}^{t}\left\{\sqrt{M_{\theta} g^{n-2}(s, a) g^{\prime}(s, a) H(t, s)} z(s)\right. \\
\left.+\frac{h(t, s)}{\sqrt{M_{\theta} g^{n-2}(s, a) g^{\prime}(s, a)}}\right\}^{2} d s \\
\quad+\int_{t_{1}}^{t} \frac{h^{2}(t, s)}{2 M_{\theta} g^{n-2}(s, a) g^{\prime}(s, a)} d s \\
\leq \quad H\left(t, t_{1}\right) z\left(t_{1}\right)+\int_{t_{1}}^{t} \frac{h^{2}(t, s)}{2 M_{\theta} g^{n-2}(s, a) g^{\prime}(s, a)} d s .
\end{gathered}
$$


Furthermore, for $t>u \geq t_{0}$, we have

$$
\begin{aligned}
& \frac{1}{H\left(t, t_{0}\right)} \int_{u}^{t}\left[\lambda H(t, s) \int_{a}^{b} p(s, \xi)\{1-c[g(s, \xi)]\} d \sigma(\xi)-\frac{h^{2}(t, s)}{2 M_{\theta} g^{n-2}(s, a) g^{\prime}(s, a)}\right] d s \\
\leq & \frac{H(t, u)}{H\left(t, t_{0}\right)} z(u) .
\end{aligned}
$$

From (17) and $\left(H_{2}\right)$, we conclude that

$$
\begin{aligned}
\varphi(u) \leq & \frac{1}{H\left(t, t_{0}\right)} \int_{u}^{t}\left[\lambda H(t, s) \int_{a}^{b} p(s, \xi)\{1-c[g(s, \xi)]\} d \sigma(\xi)\right. \\
& \left.-\frac{h^{2}(t, s)}{2 M_{\theta} g^{n-2}(s, a) g^{\prime}(s, a)}\right] d s \\
\leq & \frac{H(t, u)}{H\left(t, t_{0}\right)} z(u) \leq z(u),
\end{aligned}
$$

which implies that

$$
\varphi_{+}^{2}(u) \leq z^{2}(u)
$$

Let

$$
\begin{aligned}
v(t) & =\frac{1}{H\left(t, t_{0}\right)} \int_{t_{1}}^{t} \sqrt{H(t, s)} h(t, s) z(s) d s \\
w(t) & =\frac{1}{H\left(t, t_{0}\right)} \int_{t_{1}}^{t} \frac{M_{\theta}}{2} H(t, s) g^{n-2}(s, a) g^{\prime}(s, a) z^{2}(s) d s .
\end{aligned}
$$

Then, from (20), we find

$$
v(t)+w(t) \leq \frac{H\left(t, t_{1}\right)}{H\left(t, t_{0}\right)} z\left(t_{1}\right)-\frac{1}{H\left(t, t_{0}\right)} \int_{t_{1}}^{t} \lambda H(t, s) \int_{a}^{b} p(s, \xi)\{1-c[g(s, \xi)]\} d \sigma(\xi) d s .
$$

It follows from (17) that

$$
\liminf _{t \rightarrow \infty} \frac{1}{H\left(t, t_{0}\right)} \int_{u}^{t} \lambda H(t, s) \int_{a}^{b} p(s, \xi)\{1-c[g(s, \xi)]\} d \sigma(\xi) d s \geq \varphi(u) .
$$

Furthermore, we obtain

$$
\begin{aligned}
& \limsup _{t \rightarrow \infty} \frac{1}{H\left(t, t_{0}\right)} \int_{t_{1}}^{t} \lambda H(t, s) \int_{a}^{b} p(s, \xi)\{1-c[g(s, \xi)]\} d \sigma(\xi) d s \\
& -\liminf _{t \rightarrow \infty} \frac{1}{H\left(t, t_{0}\right)} \int_{t_{1}}^{t} \frac{h^{2}(t, s)}{2 M_{\theta} g^{n-2}(s, a) g^{\prime}(s, a)} d s \geq \varphi\left(t_{1}\right) .
\end{aligned}
$$

It turns out from (26) and (16)

$$
\liminf _{t \rightarrow \infty} \frac{1}{H\left(t, t_{0}\right)} \int_{t_{1}}^{t} \frac{h^{2}(t, s)}{2 M_{\theta} g^{n-2}(s, a) g^{\prime}(s, a)} d s<\infty .
$$


Thus, there exists a sequence $\left\{t_{n}\right\}_{1}^{\infty}$ in $\left[t_{1}, \infty\right)$ such that $\lim _{n \rightarrow \infty} t_{n}=\infty$ that satisfies

$$
\lim _{n \rightarrow \infty} \frac{1}{H\left(t_{n}, t_{0}\right)} \int_{t_{1}}^{t_{n}} \frac{h^{2}\left(t_{n}, s\right)}{2 M_{\theta} g^{n-2}(s, a) g^{\prime}(s, a)} d s<\infty .
$$

Result (27) implies that

$$
\begin{aligned}
\limsup _{t \rightarrow \infty}\{v(t)+w(t)\} & \\
& \leq z\left(t_{1}\right)-\liminf _{t \rightarrow \infty} \frac{1}{H\left(t, t_{0}\right)} \int_{t_{1}}^{t} \lambda H(t, s) \int_{a}^{b} p(s, \xi)\{1-c[g(s, \xi)]\} d \sigma(\xi) d s \\
& \leq z\left(t_{1}\right)-\varphi\left(t_{1}\right) \triangleq M
\end{aligned}
$$

Then, for any sufficiently large $n$, we have

$$
v\left(t_{n}\right)+w\left(t_{n}\right)<M_{1}
$$

where $M_{1}>M, M$ and $M_{1}$ are constant. According to the definition of $w(t)$, we have

$$
w^{\prime}(t)=\int_{t_{1}}^{t} \frac{M_{\theta}\left(H_{t}^{\prime}(t, s) H\left(t, t_{0}\right)-H_{t}^{\prime}\left(t, t_{0}\right) H(t, s)\right)}{2 H^{2}\left(t, t_{0}\right)} g^{n-2}(s, a) g^{\prime}(s, a) z^{2}(s) d s .
$$

Since $H_{t}^{\prime}(t, s)$ is nondecreasing and $\left(H_{2}\right)$ holds, we have $w^{\prime}(t) \geq 0$, thus, $w(t)$ is increasing, and $\lim _{t \rightarrow \infty} w(t)=l$ exists, where $l$ is finite or infinite. In the case of $\lim _{n \rightarrow \infty} w\left(t_{n}\right)=\infty$. Consequently, it follows from (29) that

$$
\lim _{n \rightarrow \infty} v\left(t_{n}\right)=-\infty
$$

and

$$
\frac{v\left(t_{n}\right)}{w\left(t_{n}\right)}+1<\frac{M_{1}}{w\left(t_{n}\right)}
$$

Thus, for any $0<\varepsilon<1$ and sufficiently large $n$, we have

$$
\frac{v\left(t_{n}\right)}{w\left(t_{n}\right)}<\varepsilon-1<0 .
$$

On the other hand, by using the Schwartz inequality, for $t \geq t_{1}$, we obtain

$$
\begin{aligned}
0 \leq & v^{2}\left(t_{n}\right)=\frac{1}{H^{2}\left(t_{n}, t_{0}\right)}\left\{\int_{t_{1}}^{t_{n}} \sqrt{H\left(t_{n}, s\right)} h\left(t_{n}, s\right) z(s) d s\right\}^{2} \\
\leq & \left\{\frac{1}{H\left(t_{n}, t_{0}\right)} \int_{t_{1}}^{t_{n}} \frac{M_{\theta}}{2} H\left(t_{n}, s\right) g^{n-2}(s, a) g^{\prime}(s, a) z^{2}(s) d s\right\} \\
& \times\left\{\frac{1}{H\left(t_{n}, t_{0}\right)} \int_{t_{1}}^{t_{n}} \frac{2 h^{2}\left(t_{n}, s\right)}{M_{\theta} g^{n-2}(s, a) g^{\prime}(s, a)} d s\right\} \\
= & w\left(t_{n}\right) \frac{1}{H\left(t_{n}, t_{0}\right)} \int_{t_{1}}^{t_{n}} \frac{2 h^{2}\left(t_{n}, s\right)}{M_{\theta} g^{n-2}(s, a) g^{\prime}(s, a)} d s .
\end{aligned}
$$

Then,

$$
0 \leq \frac{v^{2}\left(t_{n}\right)}{w\left(t_{n}\right)} \leq \frac{1}{H\left(t_{n}, t_{0}\right)} \int_{t_{1}}^{t_{n}} \frac{2 h^{2}\left(t_{n}, s\right)}{M_{\theta} g^{n-2}(s, a) g^{\prime}(s, a)} d s
$$


It follows from (27) that

$$
0 \leq \lim _{n \rightarrow \infty} \frac{v^{2}\left(t_{n}\right)}{w\left(t_{n}\right)}<\infty
$$

In view of (31), we obtain

$$
\lim _{n \rightarrow \infty} \frac{v\left(t_{n}\right)}{w\left(t_{n}\right)}=\lim _{n \rightarrow \infty} \frac{v^{\prime}\left(t_{n}\right)}{w^{\prime}\left(t_{n}\right)} \leq \varepsilon-1<0,
$$

and then,

$$
\lim _{n \rightarrow \infty} \frac{v^{2}\left(t_{n}\right)}{w\left(t_{n}\right)}=\lim _{n \rightarrow \infty} \frac{2 v\left(t_{n}\right) v^{\prime}\left(t_{n}\right)}{w^{\prime}\left(t_{n}\right)}=2 \lim _{n \rightarrow \infty} v\left(t_{n}\right) \lim _{n \rightarrow \infty} \frac{v^{\prime}\left(t_{n}\right)}{w^{\prime}\left(t_{n}\right)}=\infty
$$

which contradicts (33). Thus, we have $\lim _{t \rightarrow \infty} w(t)=l<\infty$. Furthermore, according to (24), we conclude that

$$
\begin{gathered}
\lim _{t \rightarrow \infty} \frac{1}{H\left(t, t_{0}\right)} \int_{t_{1}}^{t} H(t, s) g^{n-2}(s, a) g^{\prime}(s, a) \varphi_{+}^{2}(s) d s \\
\leq \lim _{t \rightarrow \infty} \frac{1}{H\left(t, t_{0}\right)} \int_{t_{1}}^{t} H(t, s) g^{n-2}(s, a) g^{\prime}(s, a) z^{2}(s) d s=\frac{2}{M_{\theta}} \lim _{t \rightarrow \infty} w(t)<\infty
\end{gathered}
$$

which implies that

$$
\begin{aligned}
\lim _{t \rightarrow \infty} & \frac{1}{H\left(t, t_{0}\right)} \int_{t_{0}}^{t} H(t, s) g^{n-2}(s, a) g^{\prime}(s, a) \varphi_{+}^{2}(s) d s \\
= & \lim _{t \rightarrow \infty} \frac{1}{H\left(t, t_{0}\right)}\left[\int_{t_{0}}^{t_{1}}+\int_{t_{1}}^{t}\right] H(t, s) g^{n-2}(s, a) g^{\prime}(s, a) \varphi_{+}^{2}(s) d s \\
\leq & \int_{t_{0}}^{t_{1}} H(t, s) g^{n-2}(s, a) g^{\prime}(s, a) \varphi_{+}^{2}(s) d s+\frac{2}{M_{\theta}} \lim _{t \rightarrow \infty} w(t)<\infty .
\end{aligned}
$$

The latter contradicts (18). Therefore, the proof of Theorem 2 is complete.

\section{References}

[1] W.N.Li, B.T.Cui, A class of even order neutral differential inequalities and its applications. Appl. Math.Comput., 122(2001):95-106.

[2] X.Z.Liu, X.L.Fu, High order nonlinear differential inequalities with distributed deviating arguments and applications. Appl. Math. Comput., 98(1999):147-167.

[3] Ch.G.Philos, A new criterion for the oscillatory and asymptotic behavior of delay differential equations. Bull.Acad.Pol.Sci.Ser.Sci.Mat., 39(1981):61-64. 


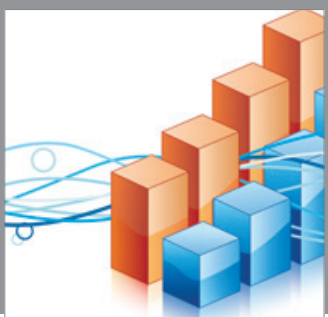

Advances in

Operations Research

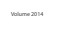

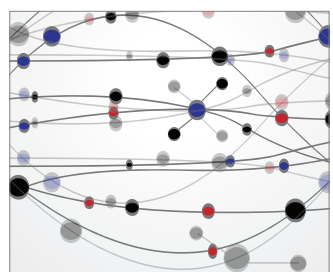

\section{The Scientific} World Journal
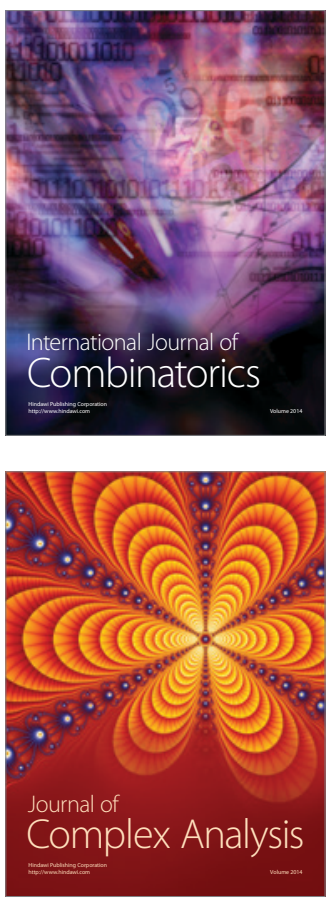

International Journal of

Mathematics and

Mathematical

Sciences
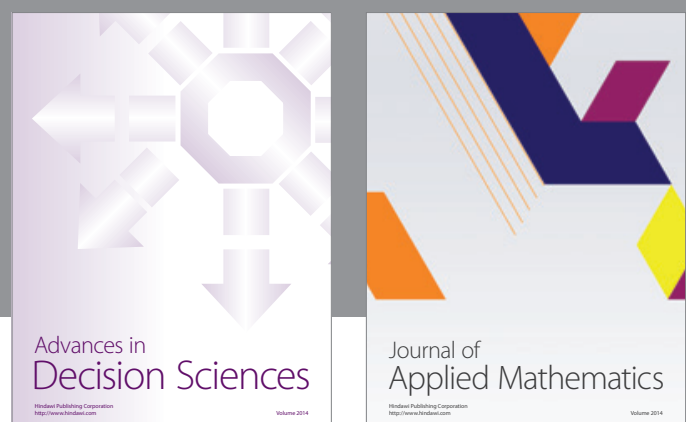

Journal of

Applied Mathematics
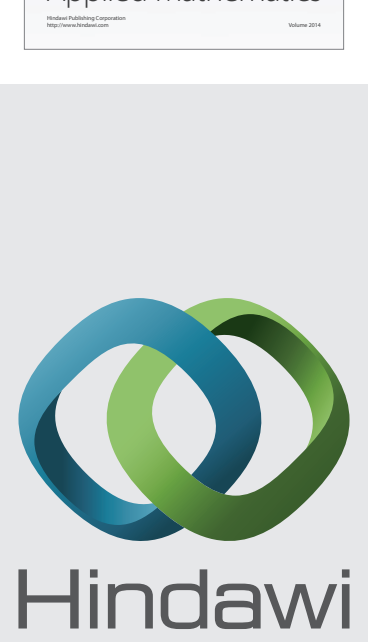

Submit your manuscripts at http://www.hindawi.com
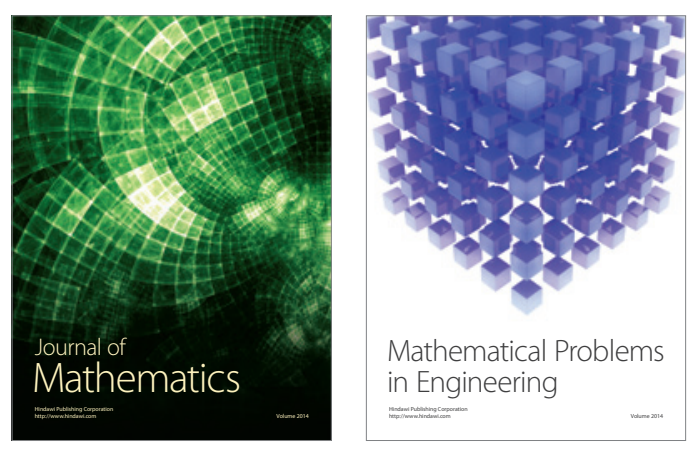

Mathematical Problems in Engineering
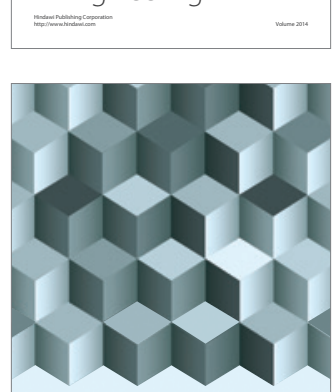

Journal of

Function Spaces
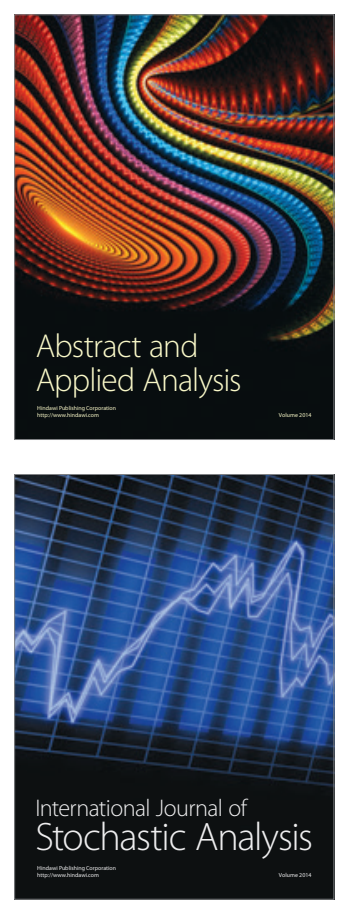

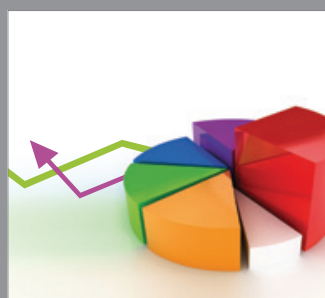

ournal of

Probability and Statistics

Promensencen
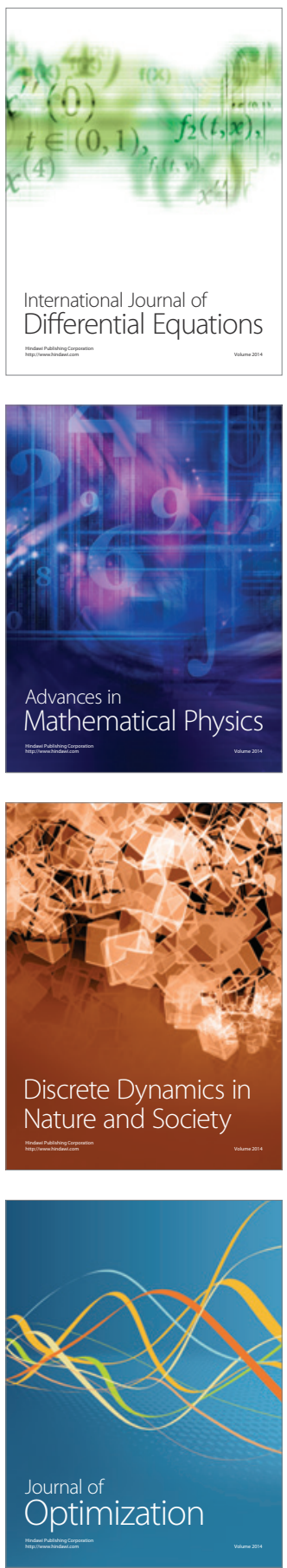